\title{
Dynamic Network Signal Processing using Latent Threshold Models
}

\author{
Jouchi Nakajima $^{1}$, Mike West ${ }^{2}$ \\ Duke University
}

\begin{abstract}
We discuss multivariate time series signal processing that exploits a recently introduced approach to dynamic sparsity modelling based on latent thresholding. This methodology induces time-varying patterns of zeros in state parameters that define both directed and undirected associations between individual time series, so generating statistical representations of the dynamic network relationships among the series. Following an overview of model contexts and Bayesian analysis for dynamic latent thresholding, we exemplify the approach in two studies: one of foreign currency exchange rate (FX) signal processing, and one in evaluating dynamics in multiple electroencephalography (EEG) signals. These studies exemplify the utility of dynamic latent threshold modelling in revealing interpretable, data-driven dynamics in patterns of network relationships in multivariate time series.
\end{abstract}

Keywords: Dynamic graphical models; EEG signal processing; Financial time series; Multivariate volatility; Sparse time-varying regression

Email addresses: jouchi.nakajima@boj.or.jp (Jouchi Nakajima ${ }^{1}$ ), mw@stat.duke.edu (Mike West ${ }^{2}$ )

${ }^{1}$ Present position and address: Deputy Director, Monetary Affairs Department, Bank of Japan, 2-1-1 Nihonbashi-Hongokucho, Chuo-ku, Tokyo 103-0021, Japan

${ }^{2}$ Corresponding author: The Arts \& Sciences Professor of Statistics \& Decision Sciences, Department of Statistical Science, Duke University, Durham 27708-0251, U.S.A. Tel: +1 9196848842 


\section{Introduction}

Dynamic network structures generating inter-related time series arise in many scientific fields-from neuroscience, to engineering signal processing, to financial econometrics, and others. For statistical definition and estimation of empirical networks, vector autoregressive (VAR) models have become standard tools (e.g. Valdés-Sosa 2004; Valdés-Sosa et al. 2005). A typical approach examines the relevance of VAR coefficients, linking to the idea of Granger (1969) causality to suggest and quantify feed-forward network connections. Time-varying VAR (TV-VAR) models refine this, in allowing for temporal changes in the strengths of such relationships (e.g. Kitagawa and Gersch 1996; Primiceri 2005; Fujita et al. 2007; Prado 2010a,b; Prado and West 2010; Koop and Korobilis 2013). Linked to this, statistical graphical models are becoming increasingly popular in defining models of potentially sparse contemporaneous network associations induced by patterns of conditional independence (Whittaker 1990; Lauritzen 1996; Dobra et al. 2004; Jones et al. 2005; Carvalho and West 2007; West 2013).

This paper overviews a framework of dynamic sparsity in empirical network relationships using the latent threshold modelling (LTM) concept. Introduced in Nakajima and West (2013a), the LTM approach defines a class of parametrized models for thresholding state parameter processes in broad classes of multivariate time series models. Timeand data-adaptive thresholding can induce patterns of temporal sparsity in the resulting, practically effective state processes. We overlay the LTM method on TV-VAR models with Cholesky-style multivariate stochastic volatility (MSV) components (e.g. Lopes et al. 2012). We denote the overall model class by TV-VAR-MSV. The time-varying VAR aspects flexibly represent and quantify dynamics in the feed-forward relationships among series, while the time-varying multivariate stochastic volatility elements represent and quantify dynamics in contemporaneous relationships.

The LTM concept allows for, and induces, dynamic patterns of sparsity in both feedforward and contemporaneous networks linking the series. The model structure also allows decoupling of model fitting to a set of parallel but linked univariate dynamic models; computation scales only linearly with time series dimension as a result.

Following the introductory Section 1, we detail TV-VAR-MSV models and review the LTM ideas in Section 2. Section 3 outlines Bayesian analysis and computation for model fitting. Two applied studies follow: Section 4 presents an econometric finance example, exploring dynamic dependencies in international financial markets; Section 5 provides an example of dynamic network modelling of connectivities among multiple EEG signals from a neuropsychiatric study. Section 6 provides concluding comments.

Some notation: We denote vectors and matrices via bold font. We use the distributional notation $\boldsymbol{y} \sim N(\boldsymbol{m}, \boldsymbol{V}), d \sim U(a, b), p \sim B(a, b), v \sim G(a, b)$, for the multivariate normal, uniform, beta, and gamma distributions, respectively. We also use $s: t$ to denote $s, s+$ $1, \ldots, t$ when $s<t$, for succinct subscripting; e.g., $\boldsymbol{y}_{1: T}$ denotes $\left\{\boldsymbol{y}_{1}, \ldots, \boldsymbol{y}_{T}\right\}$. 


\section{Latent Threshold TV-VAR-MSV Models}

\subsection{TV-VAR Modelling}

For the $m \times 1$-vector time series $\boldsymbol{y}_{t},(t=1,2, \ldots)$, consider the $\operatorname{TV} \operatorname{VAR}(p)$ model

$$
\boldsymbol{y}_{t}=\sum_{j=1}^{p} \boldsymbol{\Gamma}_{j t} \boldsymbol{y}_{t-j}+\boldsymbol{u}_{t}, \quad \boldsymbol{u}_{t} \sim N\left(\mathbf{0}, \boldsymbol{\Sigma}_{t}\right)
$$

where $\boldsymbol{\Gamma}_{j t}$ is the $m \times m$ matrix of time-varying coefficients at lag $j,(j=1: p)$, and $\boldsymbol{\Sigma}_{t}$ the variance matrix of the time $t$ innovations vector $\boldsymbol{u}_{t}$. The model can easily be extended to include time-varying intercepts and dynamic regressions on other exogenous predictors known at time $t$, but that is not of main interest here.

Denote by $\Omega_{t}$ the time-varying precision matrix $\Omega_{t}=\Sigma_{t}^{-1}$. Using Cholesky decomposition we can write

$$
\boldsymbol{\Sigma}_{t}=\boldsymbol{A}_{t}^{-1} \boldsymbol{\Lambda}_{t}\left(\boldsymbol{A}_{t}^{\prime}\right)^{-1}, \quad \text { equivalently } \boldsymbol{\Omega}_{t}=\boldsymbol{A}_{t}^{\prime} \boldsymbol{\Lambda}_{t}^{-1} \boldsymbol{A}_{t},
$$

with

$$
\boldsymbol{A}_{t}=\left(\begin{array}{cccc}
1 & 0 & \cdots & 0 \\
-a_{21, t} & \ddots & \ddots & \vdots \\
\vdots & \ddots & \ddots & 0 \\
-a_{m 1, t} & \cdots & -a_{m, m-1, t} & 1
\end{array}\right) \quad \text { and } \quad \boldsymbol{\Lambda}_{t}=\left(\begin{array}{cccc}
\lambda_{1 t} & 0 & \cdots & 0 \\
0 & \ddots & \ddots & \vdots \\
\vdots & \ddots & \ddots & 0 \\
0 & \cdots & 0 & \lambda_{m t}
\end{array}\right)
$$

where the $a_{i j t}$ elements are real-valued and the $\lambda_{j t}$ are positive. Decompositions of this form are increasingly popular in multiple time series modelling (e.g. Pinheiro and Bates 1996; Smith and Kohn 2002; Primiceri 2005; Lopes et al. 2012; Nakajima and West 2013a). The decomposition allows for flexibility in modelling volatility matrices over time through models on the elements $a_{i j t}, \lambda_{j t}$, while also enabling efficient computation, as we detail further below.

Relating to graphical models (Whittaker 1990; Lauritzen 1996; Jones et al. 2005), a zero in the $i, j$ off-diagonal element of $\Omega_{t}$ relates to conditional independence of the corresponding innovation elements $u_{i t}, u_{j t}$ in $\boldsymbol{u}_{t}$. The undirected graph on $m$ nodes representing the $m$ scalar elements of $\boldsymbol{u}_{t}$ has edges between only those pairs of nodes that are conditionally dependent, with the missing edges corresponding to node pairs with zero precision elements. Increased levels of conditional independence are key idea to reducing parameter dimension, inducing parsimonious structure in $\Omega_{t}$, and hence $\Sigma_{t}$, as a result. Any pattern of off-diagonal zeros then represents the network of contemporaneous dependencies at time $t$. Note that many zeros among the elements $a_{i j t}$ of the lower-triangular matrix $\boldsymbol{A}_{t}$ can induce off-diagonal zeros in $\boldsymbol{\Omega}_{t}$, i.e. sparsity of $\boldsymbol{A}_{t}$ can lead to a sparse set of contemporaneous network connectivities. 


\subsection{Decoupled System of Dynamic Regressions}

From eqns. $(2,3)$ we see that

$$
\boldsymbol{A}_{t} \boldsymbol{y}_{t}=\sum_{j=1}^{p} \boldsymbol{B}_{j t} \boldsymbol{y}_{t-j}+\varepsilon_{t}, \quad \varepsilon_{t} \sim N\left(\mathbf{0}, \boldsymbol{\Lambda}_{t}\right)
$$

where: $\boldsymbol{B}_{j t}=\boldsymbol{A}_{t} \boldsymbol{\Gamma}_{j t},(j=1: p)$, and $\varepsilon_{t}=\boldsymbol{A}_{t} \boldsymbol{u}_{t}=\left(\varepsilon_{1 t}, \ldots, \varepsilon_{m t}\right)^{\prime}$ has independent elements $\varepsilon_{i t} \sim N\left(0, \lambda_{i t}\right),(i=1: m)$. The model can then be recast as the triangular system of univariate dynamic regressions

$$
\begin{aligned}
y_{1 t} & =\boldsymbol{z}_{t-1}^{\prime} \boldsymbol{a}_{1 t}+\varepsilon_{1 t}, \\
y_{2 t} & =\boldsymbol{z}_{t-1}^{\prime} \boldsymbol{a}_{2 t}+a_{21, t} y_{1 t}+\varepsilon_{2 t}, \\
y_{3 t} & =\boldsymbol{z}_{t-1}^{\prime} \boldsymbol{a}_{3 t}+a_{31, t} y_{1 t}+a_{32, t} y_{2 t}+\varepsilon_{3 t}, \\
& \vdots \\
y_{m t} & =\boldsymbol{z}_{t-1}^{\prime} \boldsymbol{a}_{m t}+a_{m 1, t} y_{1 t}+\cdots+a_{m, m-1, t} y_{m-1, t}+\varepsilon_{m t},
\end{aligned}
$$

where $\boldsymbol{z}_{t-1}=\left(\boldsymbol{y}_{t-1}^{\prime}, \ldots, \boldsymbol{y}_{t-p}^{\prime}\right)^{\prime}$ and, for each $i=1: m$, the $\boldsymbol{a}_{i t}$ is a $m p \times 1$ vector formed by vertically stacking the transposed $i$-th rows of each of $\boldsymbol{B}_{1 t}, \boldsymbol{B}_{2 t}, \ldots, \boldsymbol{B}_{p t}$ in that order.

Finally, define $\boldsymbol{x}_{1 t}=\boldsymbol{z}_{t-1}, \boldsymbol{b}_{1 t}=\boldsymbol{a}_{1 t}$, and, for $i=2: m, \boldsymbol{x}_{i t}=\left(\boldsymbol{z}_{t-1}^{\prime}, y_{1 t}, \ldots, y_{i-1, t}\right)^{\prime}$ and $\boldsymbol{b}_{i t}=\left(\boldsymbol{a}_{i t}^{\prime}, a_{i 1, t}, \ldots, a_{i, i-1, t}\right)^{\prime}$. Then the model notation is simplified as

$$
y_{i t}=\boldsymbol{x}_{i t}^{\prime} \boldsymbol{b}_{i t}+\varepsilon_{i t} \quad \text { with } \quad \varepsilon_{i t} \sim N\left(0, \lambda_{i t}\right), \quad(i=1: m)
$$

with noise terms $\varepsilon_{i t}$ that are independent across $i=1: \mathrm{m}$.

The system of equations (5) is the model representation adopted. For each $i$, note that: (i) the leading $m p$ elements of $\boldsymbol{b}_{i t}$ relate the time $t$ univariate signal $y_{i t}$ to the previous $p$ lagged signals on all series, so defining feed-forward network structure; while (ii) for signals $i=2: m$, the last $i-1$ elements of $\boldsymbol{b}_{i t}$ relate the $y_{i t}$ to the contemporaneous values of other series $y_{h t}$ for $h<i$; together these define the elements of $\boldsymbol{A}_{t}$ and hence, when coupled with $\Lambda_{t}$, we recover the full multivariate volatility matrix $\Sigma_{t}$. That is, this formulation coverts the goal of modelling multivariate volatility structure $\Sigma_{t}$ over time to that of a collection of dynamic regression vectors $\boldsymbol{b}_{i t}$ and scalar volatilities $\lambda_{i t}$. This transformed representation of MSV structure is also then key to decoupling the analyses. Under dynamic model structures and priors for each $\left(\boldsymbol{b}_{i t}, \lambda_{i t}\right)$ that are independent over $i=1: m$, Bayesian analysis reduces to computations that are conditionally independent across series $i$ and can be implemented in parallel.

The Cholesky-style representation of MSV structures that the general formulation here represents has become increasingly popular as a general approach due to (i) the decoupling that yields computational tractability, and (ii) the opportunity to model the resulting components $\left(\boldsymbol{b}_{i t}, \lambda_{i t}\right)$ in various ways. On the latter point, the entire focus of our work here is to apply latent threshold models to the $\boldsymbol{b}_{i t}$ and one of two standard univariate volatility models to the $\lambda_{i t}$, as detailed below. Connections with prior work on multivariate volatility 
include well-known latent factor models that structure $\Sigma_{t}$ via various forms of latent factor representation (e.g. Aguilar et al. 1999; Pitt and Shephard 1999; Aguilar and West 2000; Chib et al. 2006; Lopes and Carvalho 2007; Yoshida and West 2010). There are similarities with our framework, in that equation (3) is a form of (full-rank) factor decomposition in which $\boldsymbol{A}_{t}$ is the inverse of a time-varying factor loadings matrix. More directly and practically, we note that we can explicitly include latent factor structure in more elaborate LTMs by adding latent factor components to the right-hand side of equation (4) directly (Nakajima and West 2013b).

\subsection{Latent Threshold Structure}

The concept of using Bayesian variable selection methods to induce zeros in TV-VAR state parameters has become of interest in a number of literatures, especially signal processing in time series econometrics (e.g. Koop and Korobilis 2013). Coupled with this is the now traditional use of Bayesian graphical modelling to induce zeros in precision matrices of innovations in TV-VAR and other dynamic models (e.g. Carvalho and West 2007; Wang and West 2009; Wang 2010). The concept and resulting methodology of latent threshold modelling is a very general, and widely applicable strategy that permits the existence of relationships to vary over time, i.e., allowing time- and data-adaptive dynamics in sparsity patterns in all model components within an overall model structure.

We follow Nakajima and West (2013a) in applying dynamic latent thresholding to the model state vectors $\boldsymbol{b}_{1: m, t}$ in eqn. (5). We use the simplest, practicable state models, based on underlying latent autoregressive models of order one, or AR(1). For each $i=1: m$, denote the state vector elements at time $t$ by, generally, $\boldsymbol{b}_{i t}=\left(b_{i 1 t}, \ldots, b_{i k_{i}}\right)^{\prime}$, where $k_{i}=$ $m p+i-1$. For each $i=1: m, j=1: k_{i}$, we assume the univariate elements $b_{i j t}$ are generated from underlying, independent and stationary AR(1) processes $\beta_{i j t}$ as follows: for $t=1,2, \ldots$,

$$
\begin{aligned}
& b_{i j t}=\beta_{i j t} s_{i j t} \quad \text { with } \quad s_{i j t}=I\left(\left|\beta_{i j t}\right| \geq d_{i j}\right), \\
& \beta_{i j t}=\mu_{i j}+\phi_{i j}\left(\beta_{i j, t-1}-\mu_{i j}\right)+\eta_{i j t}, \quad \eta_{i j t} \sim N\left(0, v_{i j}\right),
\end{aligned}
$$

where $I(\cdot)$ is the indicator function, each $d_{i j}>0$ is a latent threshold, $\eta_{i j t}$ are independent innovations, and the three parameters $\left(\mu_{i j}, \phi_{i j}, v_{i j}\right)$ are the stationary mean, first-order autocorrelation and innovations variance of the AR(1) process $\beta_{i j t}$. For stationarity, $\left|\phi_{i j}\right|<$ 1 , while for practicality- aiming to typically reflect slow, smoothly varying states- each $\phi_{i j}$ will be expected to be positive and closer to one. Priors over these AR parameters and thresholds, coupled with priors over the volatilities $\lambda_{i t}$ in eqn. (5), will then complete the model specification.

The key LTM concept exemplified here is that each of the state coefficients is zeroed out of the corresponding model equation over any time periods for which its absolute value falls below a threshold. The shrinkage region $\left(-d_{i j}, d_{i j}\right)$ defines temporal variable selection; the corresponding conditional predictor element $x_{i j t}$ in $\boldsymbol{x}_{i t}$ plays a role in predicting $y_{i t}$ only when the associated latent $\beta_{i j t}$ is "large enough". This highlights the dynamic 
sparsity- and dynamic variable selection- induced by the construction: some state elements may be thresholded to zero for long periods of time, others not at all, while some may come into, and go out of the model as time evolves.

\subsection{Univariate Volatility Models}

For the residual volatilities $\lambda_{i t},(i=1: m)$, there are a number of candidate dynamic models. Our examples use the two central models, based on their use in prior applied work in the two areas- FX and EEG studies, respectively. Combined with the LT-MSV model components, the resulting $\Sigma_{t}$ follows a latent thresholded extension of Cholesky-type multivariate stochastic volatility models (Lopes et al. 2012; Nakajima and West 2013a).

For analysis of financial time series in Section 4, we adopt log-AR(1) stochastic volatility process models that are quite standard in finance and econometrics (e.g. Jacquier et al. 1994; Kim et al. 1998; Aguilar and West 2000; Omori et al. 2007; Prado and West 2010, chap. 7). These models take $\log$-volatilities $\delta_{i t}=\log \left(\lambda_{i t}\right)$ as independent, stationary AR(1) processes; for $i=1: m$,

$$
\delta_{i t}=\mu_{\delta i}+\phi_{\delta i}\left(\delta_{i, t-1}-\mu_{\delta i}\right)+\eta_{\delta i t}, \quad \eta_{\delta i t} \sim N\left(0, v_{\delta i}\right),
$$

with $0<\phi_{\delta i}<<1$ and innovations sequences $\eta_{\delta i t}$ independent over time and across $i$. The triples $\left(\mu_{\delta i}, \phi_{\delta i}, v_{\delta i}\right)$ are hyperparameters to be estimated.

For analysis of EEG signals in Section 5- where forecasting is of little interest, while adapting to substantial stochastic volatility over time is key- we adopt the traditional multiplicative random-walk model of univariate volatilities based on variance discounting. This relates to prior studies of EEG data using alternative state-space models but exploiting this model of volatilities (e.g. West et al. 1999; Prado et al. 2001; Prado and West 2010). Here the $\delta_{i t}$ evolve as $\delta_{i t}=\delta_{i, t-1} \rho_{i t}$ where $\rho_{i t}$ are positive innovations with scaled beta distributions (conditionally independent over $t$ and independent across $i$ ). Volatility fluctuations over time in this univariate gamma-beta random walk model is defined by a single discount factor, specified as a model tuning parameter (West and Harrison 1997, chap. 10; Prado and West 2010, chap. 4).

\section{Priors and Posterior Computation}

Bayesian analysis uses Markov chain Monte Carlo (MCMC) methods, extending traditional posterior sampling methods for dynamic regression models (e.g. West and Harrison 1997; Prado and West 2010) to incorporate the latent threshold structure. Additional steps use standard Metropolis Hastings algorithms to generate sequences of the latent $\beta_{i j t}$ processes over time, and for the associated latent thresholds $d_{i j}$.

In the $i$-th model of eqn. (5) over a time period $t=1: T$, define:

- The set of dynamic state vectors $\boldsymbol{\beta}_{i, 1: T}=\left\{\boldsymbol{\beta}_{i 1}, \ldots, \boldsymbol{\beta}_{i T}\right\}$ where $\boldsymbol{\beta}_{i t}=\left(\beta_{i 1 t}, \ldots \beta_{i k_{i}}\right)^{\prime}$;

- Predictors $\boldsymbol{x}_{i, 1: T}=\left\{\boldsymbol{x}_{i 1}, \ldots, \boldsymbol{x}_{i T}\right\}$, with each $\boldsymbol{x}_{i t}$ being known at time $t$;

- Hyper-parameters of the component univariate AR(1) models in the LTMs for states, $\boldsymbol{\theta}_{i}=\left\{\mu_{i j}, \phi_{i j}, v_{i j} ; j=1: k_{i}\right\}$ 
- Latent thresholds, $d_{i, 1: k_{i}}=\left\{d_{i j} ; j=1: k_{i}\right\}$;

- Log volatilities $\delta_{i, 1: T}$ and associated hyper-parameters $\boldsymbol{\vartheta}_{i}=\left\{\mu_{\delta, i}, \phi_{\delta, i}, v_{\delta, i}\right\}$.

We observe data $\left\{y_{i, 1: T}, \boldsymbol{x}_{i, 1: T}\right\}$ and use MCMC methods to iteratively simulate all elements of the full posterior $p\left(\boldsymbol{\beta}_{i, 1: T}, \boldsymbol{\theta}_{i}, d_{i, 1: k_{i}}, \delta_{i, 1: T}, \boldsymbol{\vartheta}_{i} \mid y_{i, 1: T}, \boldsymbol{x}_{i, 1: T}\right)$. Key analysis components are now noted; see Appendix A of Nakajima and West (2013a) for full description of the posterior computations and relevant software.

(i) Dynamic states $\boldsymbol{\beta}_{i, 1: T}$ : Conditional on model hyper-parameters, volatility process states and data, a direct Metropolis-within-Gibbs sampling strategy simulates $\boldsymbol{\beta}_{i, 1: T}$. This sequences through each $t$, using a Metropolis-Hastings ( $\mathrm{MH}$ ) sampler for each $\boldsymbol{\beta}_{i t}$ given $\boldsymbol{\beta}_{i,-t}=\boldsymbol{\beta}_{i, 1: T} \backslash \boldsymbol{\beta}_{i t}$. This draws a candidate $\boldsymbol{\beta}_{i t}$ from the underlying non-threshold model obtained by ignoring the latent threshold, i.e., setting each $s_{i j t}=1$; the proposal distribution is simply a normal conditional posterior from the underlying conditionally linear, Gaussian dynamic model. The MH algorithm is completed by accepting the proposal with the relevant accept/reject probability (Nakajima and West 2013a). Analysis sequences through this process over $t=1: T$ to resample the entire sequence of $\boldsymbol{\beta}_{i t}$ vectors.

(ii) AR hyper-parameters $\boldsymbol{\theta}_{i}$ : With prior independence across $i=1: m$ and $j=1: k_{i}$, traditional priors for $\left(\mu_{i j}, \phi_{i j}, v_{i j}\right)$ yield easy simulation. We use a normal prior for each $\mu_{i j}$, a shifted beta prior for each $\phi_{i j}$, and an inverse gamma prior for each $v_{i j}$. Resulting conditional posteriors are sampled directly or via Metropolis-Hasting accept/reject steps.

(iii) Latent threshold parameters $d_{i, 1: k_{i}}$ : Following Nakajima and West (2013a), priors are taken as $d_{i j} \sim U\left(0,\left|\mu_{i j}\right|+K w_{i j}^{1 / 2}\right)$ independently; that is, uniform priors on scales based on the variances $w_{i j}=v_{i j} /\left(1-\phi_{i j}^{2}\right)$ of the stationary marginal distribution of the underlying latent processes $\beta_{i j}$. Here $K$ is fixed at a value in the range of $3-5$. Nakajima and West (2013a) discuss the rationale for this prior specification, and the observed robustness to varying $K$ across the $3-5$ range in a range of practical studies. We discuss and detail this further in the Supplemental Material accompanying the current paper; see supplemental Section A.

In posterior computations, new threshold values are simulated, conditionally independently over $i, j$, using direct Metropolis-Hastings with candidates drawn from the conditionally uniform priors.

(iv) Volatility models (a)- $\log \operatorname{AR}(1)$ processes and parameters $\left(\delta_{i, 1: T}, \boldsymbol{\vartheta}_{i}\right)$ : Sampling conditional posteriors of $\delta_{i, 1: T}$ in the log-AR(1) models uses the standard, widely used MCMC strategy for univariate stochastic volatility (e.g. Shephard and Pitt 1997; Kim et al. 1998; Watanabe and Omori 2004; Omori et al. 2007). The associated AR(1) parameters $\boldsymbol{\vartheta}_{i}$ are assigned priors, and sampled with MCMC steps, just as for the $\boldsymbol{\theta}_{i}$ in step (ii) above.

(v) Volatility models (b)- discount volatilities $\delta_{i, 1: T}$ : In the variance discount model for volatilities, the $\delta_{i t}$ evolve over time according to $\delta_{i t}=\delta_{i, t-1} \rho_{i t}$ where $\rho_{i t}$ are positive innovations with scaled beta distributions, conditionally independent over time and independent 
across $i$. The beta distributions depend on a single discount factor that controls the variation in $\eta_{i t}$ and hence the levels of volatility in the $\delta_{i t}$ process (West and Harrison 1997, chap. 10; Prado and West 2010, chap. 4).

Posterior analysis is performed via analytically trivial forward-filtering and backward sampling. This generates samples of the full sequence $\delta_{i, 1: T}$ from exact conditional posteriors, given all other model parameters and states (e.g. Prado and West 2010, chap. 4).

As noted, these MCMC analyses are decoupled and performed in parallel over $i=1: m$. Following this, the posterior samples of $\left\{\boldsymbol{\beta}_{1: m, 1: T}, \delta_{1: m, 1: T}\right\}$ can be combined to computeby trivial transformations- the implied posterior samples of the volatility matrices $\Omega_{1: T}$ (and hence, if desired, $\boldsymbol{\Sigma}_{1: T}$ ), as well as the TV-VAR coefficient matrices $\boldsymbol{B}_{1: p, 1: T}$ in the model form of eqn. (1) (and, if desired, the $\Gamma_{1: p, 1: T}$ of eqn. (4)). This directly generates posterior inferences on the dynamics in sparsity patterns as well as quantified network relationships over time. In contexts where forecasting is also of interest, posterior predictive simulations can be trivially computed by moving sequentially through the set of triangular dynamic regressions and stepping-ahead sequentially in time. Though forecasting is not a key interest in the current paper, it is in other applications of LTMs, as demonstrated- with full computational details- in Nakajima and West (2013a,b) and Zhou et al. (2014).

\section{Application: FX Time Series Networks}

The first study applies the LTM-based network model to a series of daily foreign exchange (FX) rate returns from world-wide international financial markets. Our focus here is sparsity patterns and time-varying relationships underlying the FX series and induced dynamic networks among international financial markets. In terms of prior literature, correlation-based network analysis has previously been discussed for studying relations among stock prices (e.g. Vandewalle et al. 2001; Onnela et al. 2004). Also, Tse et al. (2010) give examples of describing empirical financial networks in which nodes are individual US stocks and edges are determined by cross-correlations of stock prices/returns and trading volumes; these authors use cut-off levels on sample correlations to delete network edges. It is well-known that correlations can mislead in this kind of study. Conditional relationships may be strong, but correlations weak; vice-versa, high correlations may result from paths in networks, rather than direct edges; and, ad-hoc correlation cutoffs are hard to rationalize on any foundational basis. Approaches anchored on cointegration models to investigate evolving patterns of interdependence among international stock markets (e.g. Awokuse et al. 2009) tend to improve on correlation-based methods, but rarely adequately reflect the volatilities in financial systems, and have no ability to formally incorporate sparsity and, especially, dynamically varying patterns of sparsity that define the empirical representations of financial networks. Global graphical model structuring has represented progress on these counts (e.g. Carvalho and West 2007; Quintana et al. 2010), but again lacks the ability to model and estimate changes over time in patterns of zeros/non-zeros in lagged coefficient matrices and/or time-varying precision matrices that define both lagged and contemporaneous networks. The current example shows the 


$\begin{array}{cclccl}1 & \text { EUR } & \text { Euro } & 11 & \text { KRW } & \text { South Korean Won } \\ 2 & \text { JPY } & \text { Japanese Yen } & 12 & \text { ZAR } & \text { South African Rand } \\ 3 & \text { GBP } & \text { British Pound Sterling } & 13 & \text { RUB } & \text { Russian Ruble } \\ 4 & \text { CHF } & \text { Swiss Franc } & 14 & \text { INR } & \text { Indian Rupee } \\ 5 & \text { AUD } & \text { Australian Dollar } & 15 & \text { BRL } & \text { Brazilian Real } \\ 6 & \text { CAD } & \text { Canadian Dollar } & 16 & \text { TWD } & \text { Taiwanese Dollar } \\ 7 & \text { SEK } & \text { Swedish Krona } & 17 & \text { THB } & \text { Thai Baht } \\ 8 & \text { NOK } & \text { Norwegian Krone } & 18 & \text { IDR } & \text { Indonesian Rupiah } \\ 9 & \text { NZD } & \text { New Zealand Dollar } & 19 & \text { CLP } & \text { Chilean Peso } \\ 10 & \text { SGD } & \text { Singapore Dollar } & 20 & \text { PHP } & \text { Philippine Peso }\end{array}$

Table 1: FX return data: 20 international currencies, ordered in decreasing order of turnover in the global foreign exchange market in April 2007 as reported by BIS (2010).

utility of latent threshold dynamic models in advancing the methodology in this field.

\subsection{Data and Priors}

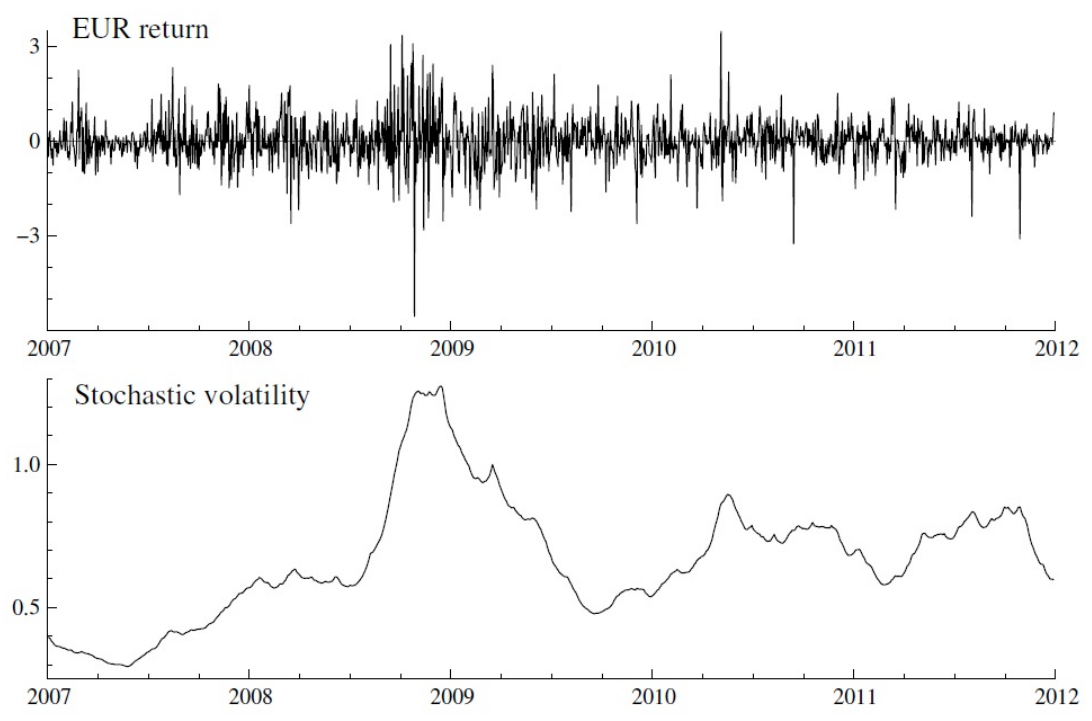

Figure 1: Euro (EUR) return series (top) and trajectory of estimated univariate stochastic volatility process (bottom), both on the \% return scale.

The data are $m=20$ daily international currency exchange rates relative to the US dollar over $T=1,304$ business days beginning in January 2007 and ending in December 2011; the currencies are listed in Table 1 . Returns are computed as $y_{i t}=100\left(p_{i t} / p_{i, t-1}-1\right)$ where $p_{i t}$ is the daily closing spot rate. Figure 1 displays a representative return series and an estimated time trajectory of its volatility from a simple univariate stochastic volatility model fitted to the single series alone- as a reference, exploratory analysis. The period includes volatile movements triggered by the global financial crisis around late 2007-09, and in 2010 related to the European sovereign (or "Euro zone") debt crisis. Across the 20 se- 

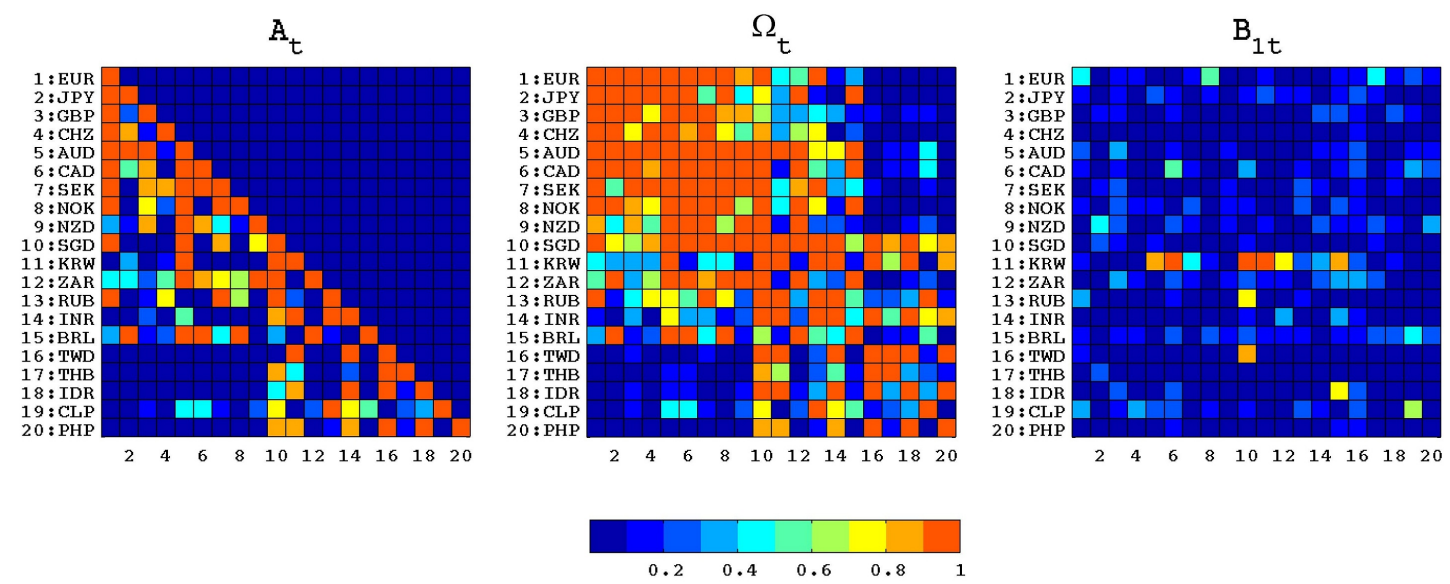

Figure 2: FX analysis- The heat-maps show: posterior probabilities of $s_{i j t}=1$ for elements of $\boldsymbol{A}_{t}$ (left); induced posterior probabilities of non-zero values in $\Omega_{t}$ representing contemporaneous network connections (center); posterior probabilities $s_{i j t}=1$ for elements of $\boldsymbol{B}_{1 t}$ (right). The panels represent inferences at the snapshot in time $t=2009 / 6 / 30$.

ries, there are marked common patterns in volatility changes as well as evident geographic region-specific, and country-specific, patterns.

In our latent threshold TV-VAR-MSV model, the currencies are ordered by the turnover in the global foreign exchange market in April 2007, as reported by BIS (2010); this gives the order in Table 1. This baseline model indicates that an idiosyncratic structural shock to one currency affects other currencies whose trade volume is less than that currency, within one business day; feedback can then occur on following days. For comparison, analysis using an alternative ordering is also examined, below.

Analysis with a TV-VAR(1) model uses priors that reflect and respect the scale of data, expected characteristics in terms of strongly persistent volatilities, and allow a wide range of potential values of underlying thresholds with rather diffuse priors. For all $i=1: m$ and $j=1: k_{i}$, we take: $1 / v_{i j} \sim G(100,0.001) ; 1 / v_{\delta i} \sim G(3,0.03) ;\left(\phi_{i j}+1\right) / 2 \sim B(20,1.5)$; $\left(\phi_{\delta i}+1\right) / 2 \sim B(20,1.5) ; \mu_{i j} \sim N(0,1)$; $\exp \left(-\mu_{\delta i}\right) \sim G(3,0.03)$; and prior on $d_{i j}$ of the conditional form noted in Section 3(iii) with $K=3$. The MCMC analysis was run for a burn-in period of 10,000 samples prior to saving the following MCMC sample of size 100,000 for the summary of posterior inferences. As in prior studies (Nakajima and West 2013a,b), MCMC monitoring assessments confirm convergence and adequacy of the Monte Carlo-based posterior summaries reported. Computations were performed using custom code in Ox (Doornik 2006); the code is available to interested readers.

\subsection{Summaries of Posterior Inferences}

Figure 2 displays posterior probabilities of $s_{i j t}=1$ for all scalar entries in $\boldsymbol{A}_{t}$, and induced posterior probabilities of non-zero entries in the precision matrix $\Omega_{t}$, at $t=651$ (2009/Jun/30), the mid-time point of the sample period. Non-zeros in a row/column pair in $\Omega_{t}$ indicate current (at time $t$ ) contemporaneous connections between the corre- 
sponding two series, in the sense of conditional dependence in the time-specific graphical model. As time evolves, these sparsity probabilities vary, mapping the evolution in time of the contemporaneous network. This chosen time point is in a period of temporarily lowered volatility of the world markets- it is timed at nine months after the Lehman Brothers shock. The panel of $\Omega_{t}$ exhibits interesting patterns of sub-networks among international currencies. The posterior probabilities for a group of the first 10 currencies are evidently high, which implies strong inter-dependencies in the simultaneous shocks to major industrialized countries. The second sub-group consists of the last 10 currencies of emerging countries, exhibiting obviously sparser structure, although relevant edges are inferred among several currencies: KRW, RUB, INR, TWD, and IDR. The first five currencies in this second sub-group (i.e., 11:KRW-15:BRL) show some connections to selected currencies in the first sub-group. In contrast, the sparse block of the lower-left sub-matrix (colored in blue) indicates a broad disconnect in this contemporaneous network of the first sub-group of developed economies from the last five currencies in the second sub-group.

Posterior probabilities of $s_{i j t}=1$ for the VAR coefficients in $\boldsymbol{B}_{1 t}$ are also plotted in Figure 2 at the selected time point in mid-2009. These are considerably sparse as expected in modelling FX returns, except for some relevant coefficients in the KRW currency. These sparsity patterns are quite stable throughout the sample period. We now focus on timevariation in the MSV model structure, and links to the known, underlying financial market conditions.
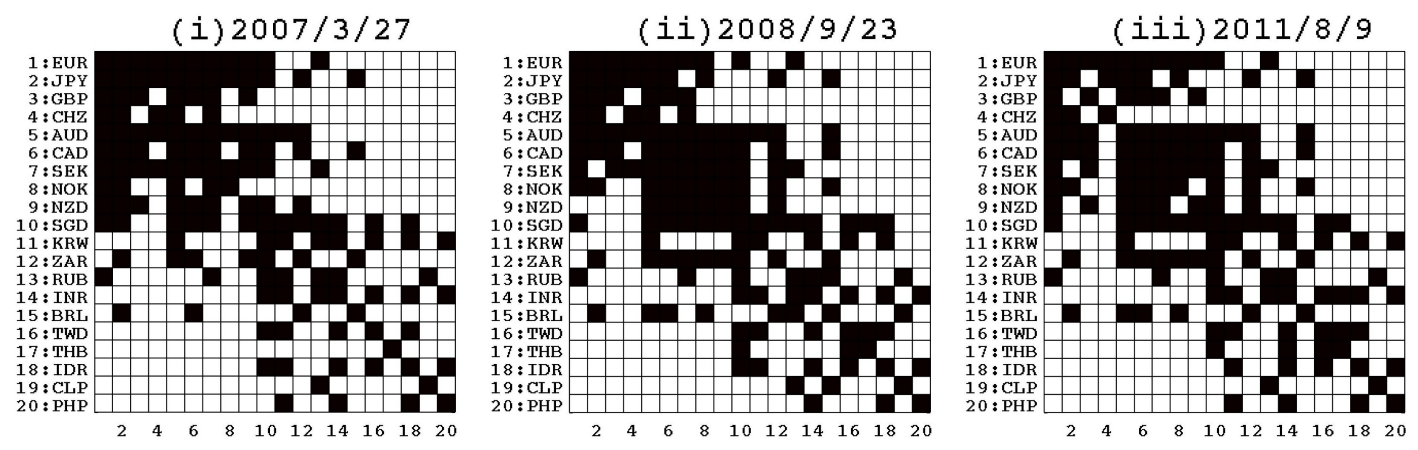

Figure 3: Evolution of contemporaneous network in FX analysis, at three selected time points. Black shading indicates posterior probabilities of at least 0.9 for non-zero values in $\Omega_{t}$ at these times.

Posterior estimates of sparsity probabilities underlying $\Omega_{t}$ over time shed light on stabilities and instabilities in inferred contemporaneous network connections. These can be viewed in connection with known global economic circumstances that relate to changes in patterns of co-movements of currency prices. Section B of the Supplemental Material provides animated displays of posterior probabilities of dynamic network connections in $\Omega_{t}$ over time. Some aspects of the changes observed can be plausibly linked to known global changes and events in financial markets. One observation is that there is a fair 
degree of stability over time, i.e., strong and sustained patterns in the network connections among these international currencies. Figure 3 shows three snapshots in time: these binary images indicate edges (non-zero precision elements, in black) for which posterior probabilities of non-zero values exceed 0.9. The first panel is at 2007/Mar/27, more than one year before the global financial crisis; this exhibits a pattern similar to that of Figure 2. The second panel shows a pattern very similar to the first, indicating many sustained links (and lack of links) in the network, but with evolving connections among some currencies during the financial crises. Interestingly, most of the industrialized countries from a sustained sub-network (the upper-left sub-matrix), while a new relevant sub-network arises in the center of the matrix around currencies (5:AUD-10:SGD) during the crisis. Turbulent fluctuations triggered by the US financial crisis spread over the world market, and most of the currencies are seemingly inter-connected in those periods, although our network model provides parsimonious structure which identifies some sub-groups of currencies. The third panel reflects further network evolution during the Euro zone debt crisis. One interesting change is that CHF has fewer edges inferred than earlier; this seems very intuitive and natural as CHF was regarded as a safe-haven currency relative to other major currencies, and therefore its exchange rate moved somewhat differently to most of the others- experiencing a historically huge appreciation- in the maturing and then ending phases of the crisis.
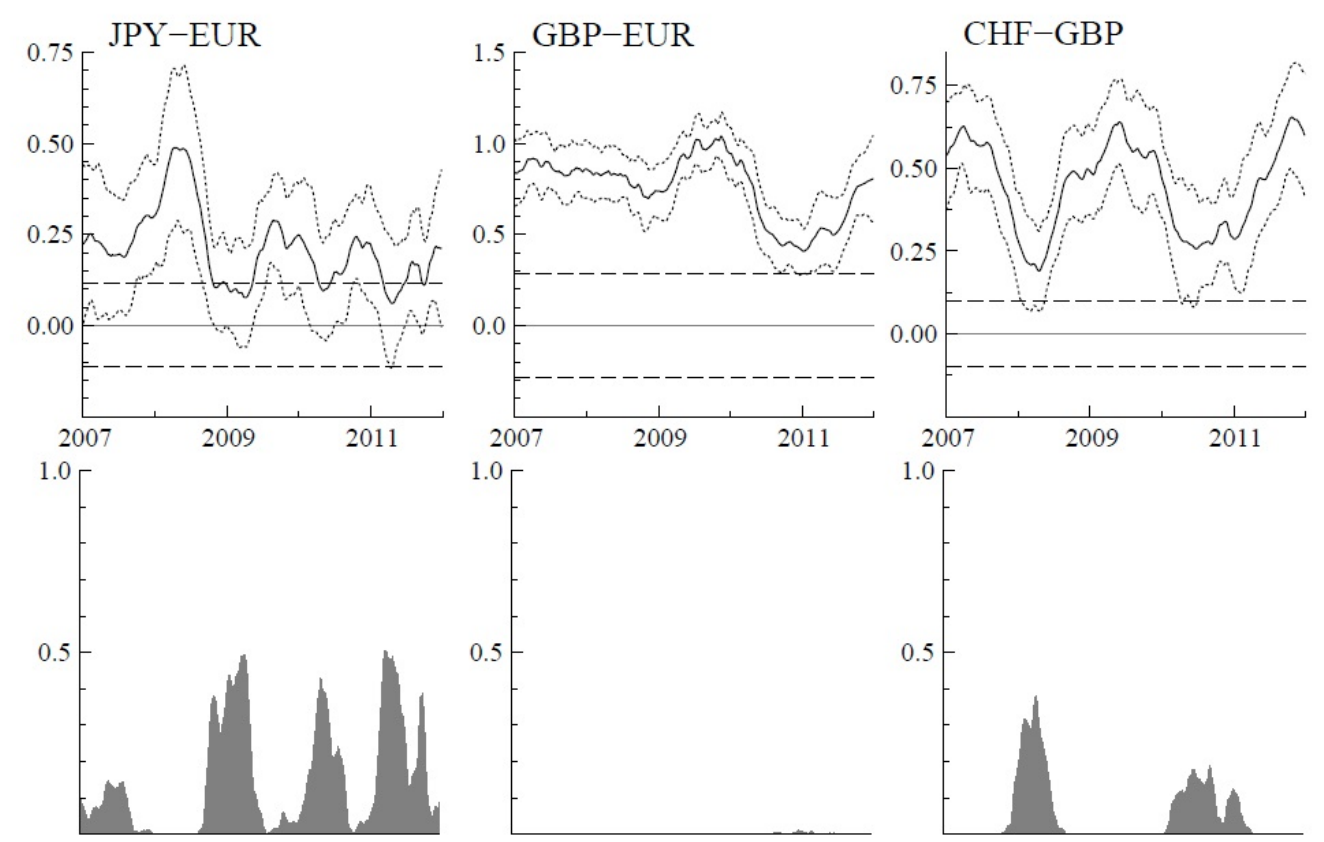

Figure 4: FX analysis- Posterior means (solid) and \pm 2 standard deviation credible intervals (dotted) of $a_{i j t}$ as well as posterior medians of latent thresholds (dashed) for selected predictors. The title line "A-B" refers to a simultaneous coefficient of currency B in predicting A contemporaneously. Posterior probabilities of $s_{i j t}=0$ are plotted below each trajectory 
We now summarize some quantified estimates of precision-related elements to add to the insights generated by sparsity probabilities alone. Figure 4 plots the time trajectories of posterior means and \pm 2 standard deviation credible intervals for three selected elements of $\boldsymbol{A}_{t}$ over the full time period. We superimpose dashed lines showing the posterior medians of the thresholds in each case (the median is a better summary for thresholds than the mean as the posteriors are typically very positively skewed and rather fat-tailed). The figure includes, below each trajectory frame, estimated sparsity probabilities for that $a_{i j t}$. The first column relates to the contemporaneous coefficient of EUR in predicting JPY. In the first phase of the global financial crisis in 2008, the coefficient rapidly declines. It is variable thereafter with temporal changes in posterior sparsity probabilities. These probabilities and coefficient trajectory indicate a relatively sustained network linkage with positive association, but one that is rather weak in magnitude throughout the turbulent economic years 2008-2012. The central column relates to the contemporaneous coefficient of the EUR in predicting GBP. The probability of a non-thresholded link is nearly one throughout the time period, i.e., the contemporaneous network sustains a link from EUR to GBP over all economic times, The upper frame shows a naturally positive coefficient; however, during 2010 this decays very substantially, presumably reflecting the weakening of co-movement and the relative strengthening of the GPB as the Euro zone experienced the debt crisis. In late 2011/early 2012 the relationship strengthened again with increasing stability in the Euro zone. Another example is the CHF-GBP link in the right-hand column of two figures. Here we can plausibly link the significant decay to much lower values during late 2007 and through 2008 as reflecting the role of CHF as a relative savehaven currency; then after some period of increased economic stability with strengthened linkage, the coefficient again decays substantially, plausibly again reflecting a safe-haven effect triggered by- and continuing through- the Euro zone crisis. Again it is worth stressing that, even if the posterior probability of a network connection is high and relatively stable, the model yields time-varying values in the state parameters that can sometimes be linked to naturally interpretable underlying financial economic circumstances.

As Supplemental Material-and as embedded animations in the original technical report version of this paper- we further illustrate and exhibit the above discussion with a video comprising a long series of frames of heat-maps of sparsity probabilities over the full course of the time series. This animates sparsity probabilities for the TV-VAR coefficients in $\boldsymbol{B}_{1 t}$, and for the elements of $\boldsymbol{A}_{t}$, as well as induced posterior probabilities of dynamic network connections in $\Omega_{t}$. Some of the above discussion of changes over time linked to known global changes and events in financial markets are really well-highlighted in these dynamic displays of temporal changes in network structures.

Finally, regarding the ordering of the time series variables in the triangular regressions, we examine the reverse ordering in this study: the FX series are reordered by lower turnovers. Figure 5 shows the posterior probabilities in $\Omega_{t}$ and $\boldsymbol{B}_{1 t}$ for the time point of 2010/Oct/19 from the baseline ordering and the reverse ordering. The currencies are reordered in the latter panels purely to enable visual comparisons. The reverse ordering 

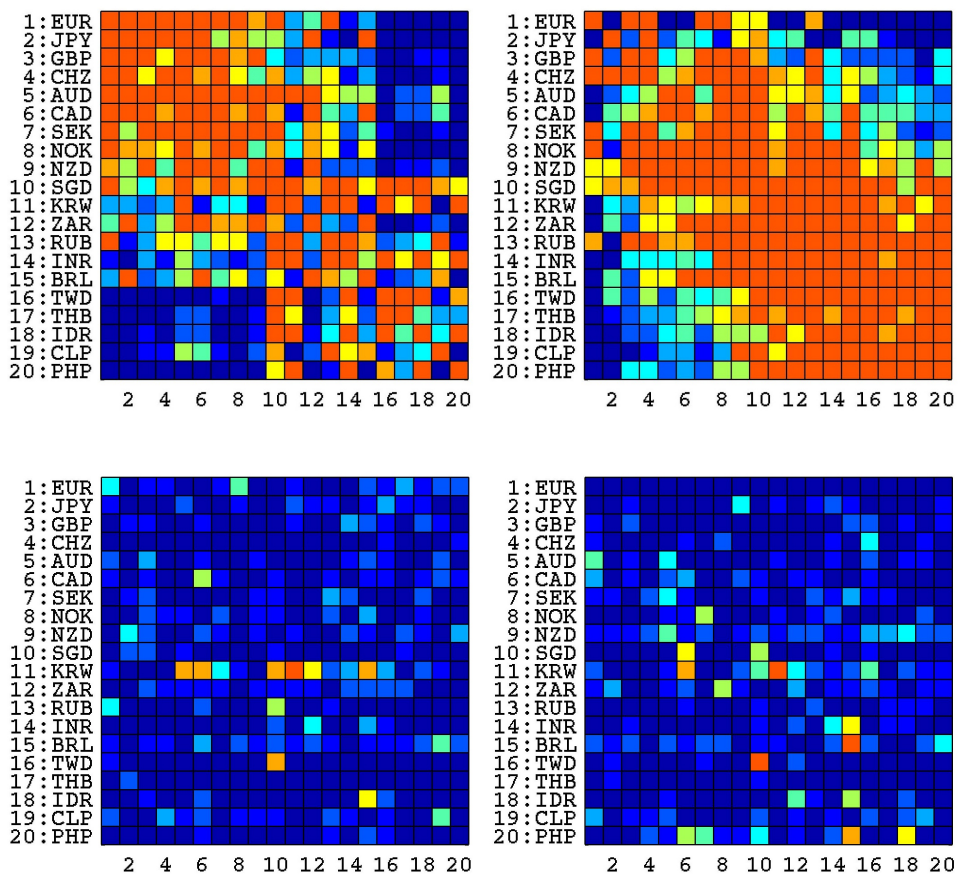

Figure 5: FX analysis- The heat-maps show: Posterior probabilities of $s_{i j t}=1$ for induced dynamic network in $\boldsymbol{\Omega}_{t}$ (top) and $\boldsymbol{B}_{1 t}$ (bottom) from baseline ordering (left) and reverse ordering (right). The panels are plotted for $t=2010 / 10 / 19$. See Figure 2 for heat-map scale.

exhibits a somewhat less sparse pattern among the industrialized countries, and somewhat sparser pattern in the emerging countries. As stated earlier, we chose the original ordering on the basis of prior expectations that the currencies of the more dominant global economies would naturally "lead" others; this is born out with the higher sparsity of the inferred dynamic networks under that ordering. Generally, an ordering inducing sparser representations is naturally to be preferred on the grounds of parsimony. To further illustrate and compare across the two variable orderings, the Supplemental Material includes additional images of sparsity probabilities for $\Omega_{t}$ at three time points: during and towards the end of the global financial crisis in 2008 and then in late 2009, and then two years later towards the end of 2011. See supplemental Section C. Again, while the general questions of variable ordering represent open research questions for future study, we do see similarities in terms of general groupings of currencies as well as in the stability of overall networks over time- the latter robust to quite variable economic conditions. Also, the lack of association between industrialized and emerging countries is robust across orderings. Additional comments on variable ordering appear in Section 6 below.

\section{Application: EEG Signal Networks}

A second study concerns analysis of network connectivities among recordings of multivariate electroencephalographic (EEG) signals. EEG recordings are measurements of electrical potential fluctuations at various scalp locations of a human subject, reflecting 


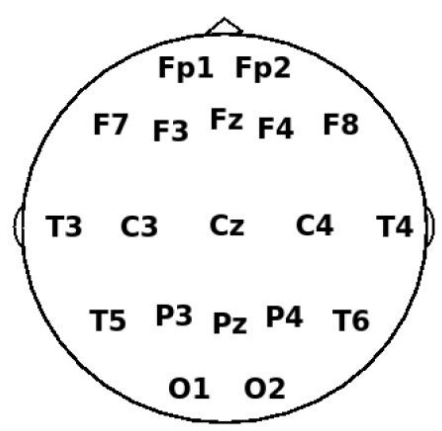

Figure 6: EEG study: Representation of approximate placement of the 19-electrodes on the scalp (looking down from above the head, which is facing upwards).

dynamics of the underlying cerebral activity as recorded indirectly on the scalp. Analysis of multichannel EEG traces has become a standard tool to understand the efficacy of electroconvulsive therapy (ECT) as a treatment for major depression. The EEG signals are generated and recorded during periods of electrically induced and controlled brain seizures in patients (e.g. Weiner and Krystal 1994). A main statistical interest here is to explore relations across EEG channels via dynamic network modelling. This builds on prior research that has popularized the use of time-varying parameter AR and VAR models with stochastic volatility (e.g., Kitagawa and Gersch 1996; West et al. 1999; Prado et al. 2001; Valdés-Sosa 2004; Valdés-Sosa et al. 2005; Prado 2010a,b; Prado and West 2010).

\subsection{Data and Priors}

This analysis uses 19-channel EEG traces recorded in one seizure of one patient, previously analyzed in part in West et al. (1999), Prado et al. (2001), and Prado and West (2010). The data are recorded in parallel from 19 electrodes of the International 10-20 EEG System, located around and over the patient's scalp; see Figure 6. The original data have sampling rate $256 \mathrm{~Hz}$ and about 26,000 observations. Following the above references, and as discussed in more detail in Prado (1998), the original series are subsampled every sixth observation after removing about 2,000 observations from the beginning (up to a higher amplitude portion of the seizure) yielding $T=3,000$ observations.

Our first analysis uses the baseline ordering of EEG locations/probes as listed in Table 2(i), starting from the vertex channel $\mathrm{Cz}$. This central channel/probe location is known as a key factor that potentially (statistically) "drives" the other EEG traces. The baseline ordering reflects this, with the order moving out clockwise from this central location, around the inner channels to the outer channels. Comparison with an alternative ordering is made later in this section.

Analysis uses the LTM-based TV-VAR-MSV model with TV-VAR order $p=2$. We examined lags up to $p=6$, finding that higher-order models yield over-fitting and considerably sparse patterns in coefficients of higher lagged predictors, while the structure of the lower lag coefficient matrices is maintained. As in the FX analysis, priors are chosen to re- 
(i) Baseline

$\begin{array}{clll}1 & \mathrm{Cz} & 11 & \mathrm{O} 1 \\ 2 & \mathrm{Pz} & 12 & \mathrm{~T} 5 \\ 3 & \mathrm{P} 3 & 13 & \mathrm{~T} 3 \\ 4 & \mathrm{C} 3 & 14 & \mathrm{~F} 7 \\ 5 & \mathrm{~F} 3 & 15 & \mathrm{Fp} 1 \\ 6 & \mathrm{Fz} & 16 & \mathrm{Fp} 2 \\ 7 & \mathrm{~F} 4 & 17 & \mathrm{~F} 8 \\ 8 & \mathrm{C} 4 & 18 & \mathrm{~T} 4 \\ 9 & \mathrm{P} 4 & 19 & \mathrm{~T} 6 \\ 10 & \mathrm{O} 2 & & \end{array}$

(ii) Factor-based

$\begin{array}{clll}1 & \mathrm{Cz} & 11 & \mathrm{~F} 3 \\ 2 & \mathrm{Pz} & 12 & \mathrm{~T} 3 \\ 3 & \mathrm{P} 3 & 13 & \mathrm{~F} 7 \\ 4 & \mathrm{~T} 5 & 14 & \mathrm{Fp} 2 \\ 5 & \mathrm{P} 4 & 15 & \mathrm{O} 2 \\ 6 & \mathrm{C} 3 & 16 & \mathrm{Fp} 1 \\ 7 & \mathrm{C} 4 & 17 & \mathrm{~F} 4 \\ 8 & \mathrm{~T} 6 & 18 & \mathrm{~F} 8 \\ 9 & \mathrm{O} 1 & 19 & \mathrm{~T} 4 \\ 10 & \mathrm{Fz} & & \end{array}$

Table 2: 19 EEG channels: (i) Baseline ordering, and (ii) Factor-based ordering.

flect and respect the scale of data, expected characteristics in terms of strongly persistent volatilities, and to allow a wide range of potential values of underlying thresholds with rather diffuse priors. For each $i=1: m$ and $j=1: k_{i}$, we take: $1 / v_{i j} \sim G\left(200,10^{-4}\right)$; $\left(\phi_{i j}+1\right) / 2 \sim B(20,1.5) ; \mu_{i j} \sim N(0,1)$; and the same structure for priors on thresholds $d_{i}$. For the time-varying volatility processes $\lambda_{i t}$, this analysis adopts the traditional multiplicative random-walk model of univariate volatilities based on variance discounting (West and Harrison 1997, chap. 10; Prado and West 2010, chap. 4). This follows the use of this volatility structure in previous works with such data (e.g. West et al. 1999; Prado et al. 2001). The volatility discount factor is set at 0.99 for each of the $m=19$ processes. Finally, MCMC burn-in and saved sample sizes are as in the FX study of the previous section.

\subsection{Summaries of Posterior Inferences}

Figure 7 displays posterior probabilities of $s_{i j t}=1$ for the scalar elements in $\boldsymbol{A}_{t}$ and induced posterior probabilities of non-zero elements- i.e., dynamic contemporaneous network connections- in $\Omega_{t}$. These are shown at the selected time $t=1,501$. The panels exhibit a notable band pattern, linked to the spatial layout of the EEG channels. Also shown are similar heat-maps of sparsity probabilities in the two TV-VAR coefficient matrices $\boldsymbol{B}_{1: 2, t}$ at the time point. We see the natural and expected increase of suggested sparsity with lag, and strong evidence of feed-forward network connectivities across sparse but non-negligible numbers of channels. We explore and visualize such structure in more detail, referring to a series of additional plots and accompanying animations.

Figure 8 shows heat maps of the posterior probabilities of non-zero entries in the $\Omega_{t}$ at the same time point as in Figure 7. Each sub-map is plotted at the location of its channel and the channel location is marked by $\otimes$ within each sub-map. The heat-maps represents (linear) interpolation of the sparsity probabilities on precision elements from channel/probe $\otimes$ to each of the other 18 channels, visually representing the contempo-

raneous connectivities at this time point. We see strong evidence of connections among 

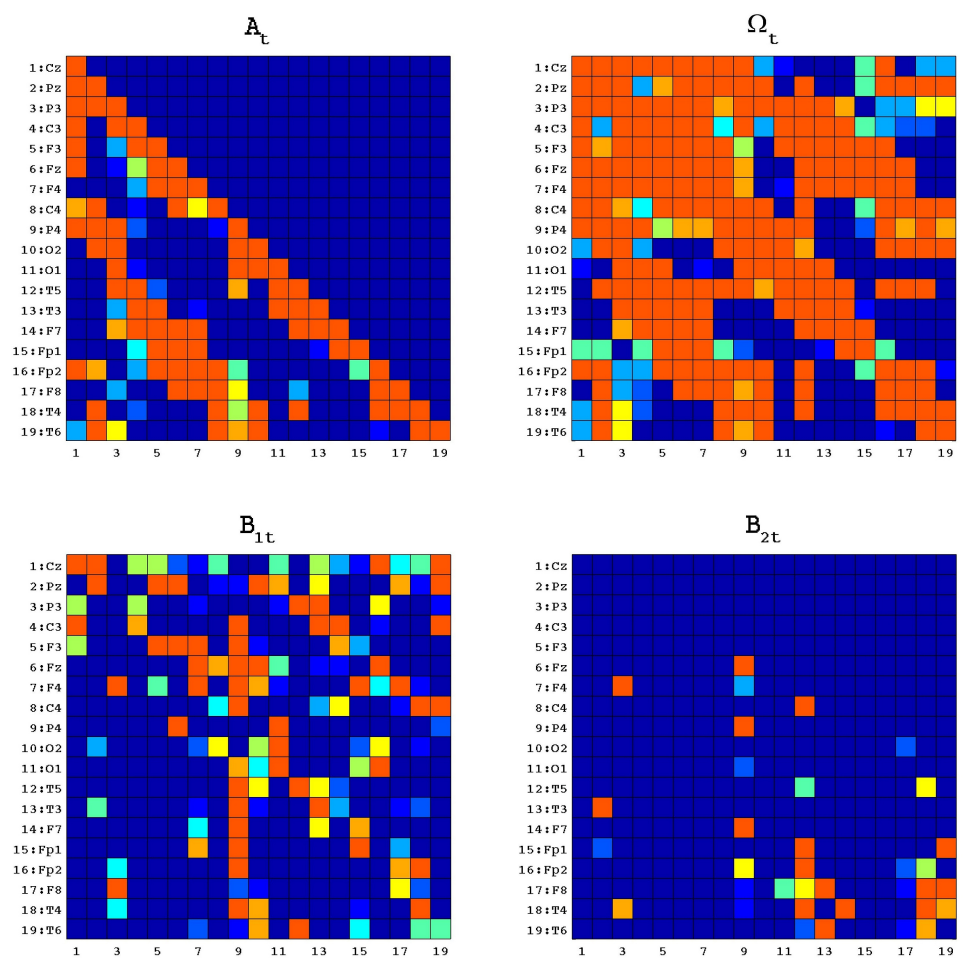

Figure 7: EEG analysis- The heat-maps show: Posterior probabilities of $s_{i j t}=1$ for elements of $\boldsymbol{A}_{t}$, induced posterior probabilities of dynamic network structure in $\Omega_{t}$, and posterior probabilities $s_{i j t}=1$ for the TV-VAR coefficients in $\boldsymbol{B}_{1: 2, t}$ at selected time snapshot $t=1,501$. See Figure 2 for heat-map scale.

geographically neighboring channels, but also evidence of more distal, non-neighboring connections in some cases. The banded sparsity pattern inferred in $\Omega_{t}$ indicates relevant spatial relations between channels in the inner circle and in the outer circle under this baseline ordering. The posterior estimates exhibit notable asymmetry between frontal and occipital regions, as well as left and right hand sides; this echoes empirical findings under previous models that link to known/expected patterns in EEG waveforms around the brain through the course of an induced siezure (Prado et al. 2001). In the prefrontal region, Fp1 is simultaneously related only to neighboring frontal channels, while FP2 is related to channels over the central and even to the occipital channel in the right-hand side. Interestingly, the frontal channels, such as F7, F3, FZ, and F4, are typically conditionally independent of the parietal and occipital right sites. For temporal channels, T3 is connected to neighboring frontal region. In contrast, T4 exhibits relations with the neighboring parietal and occipital regions up to T5. T5 is linked with a wide range of regions except $\mathrm{Cz}$, although $\mathrm{T} 6$ is connected only with a few neighboring channels.

These characteristics of networks among the EEG channels are dynamic, in part. Figure 9 shows an evolution of the posterior sparsity probabilities for $\Omega_{t}$ and the induced spatially interpolated maps for three selected channels at four time points representing early, mid, later and end periods of the time series. High posterior probabilities for edges 


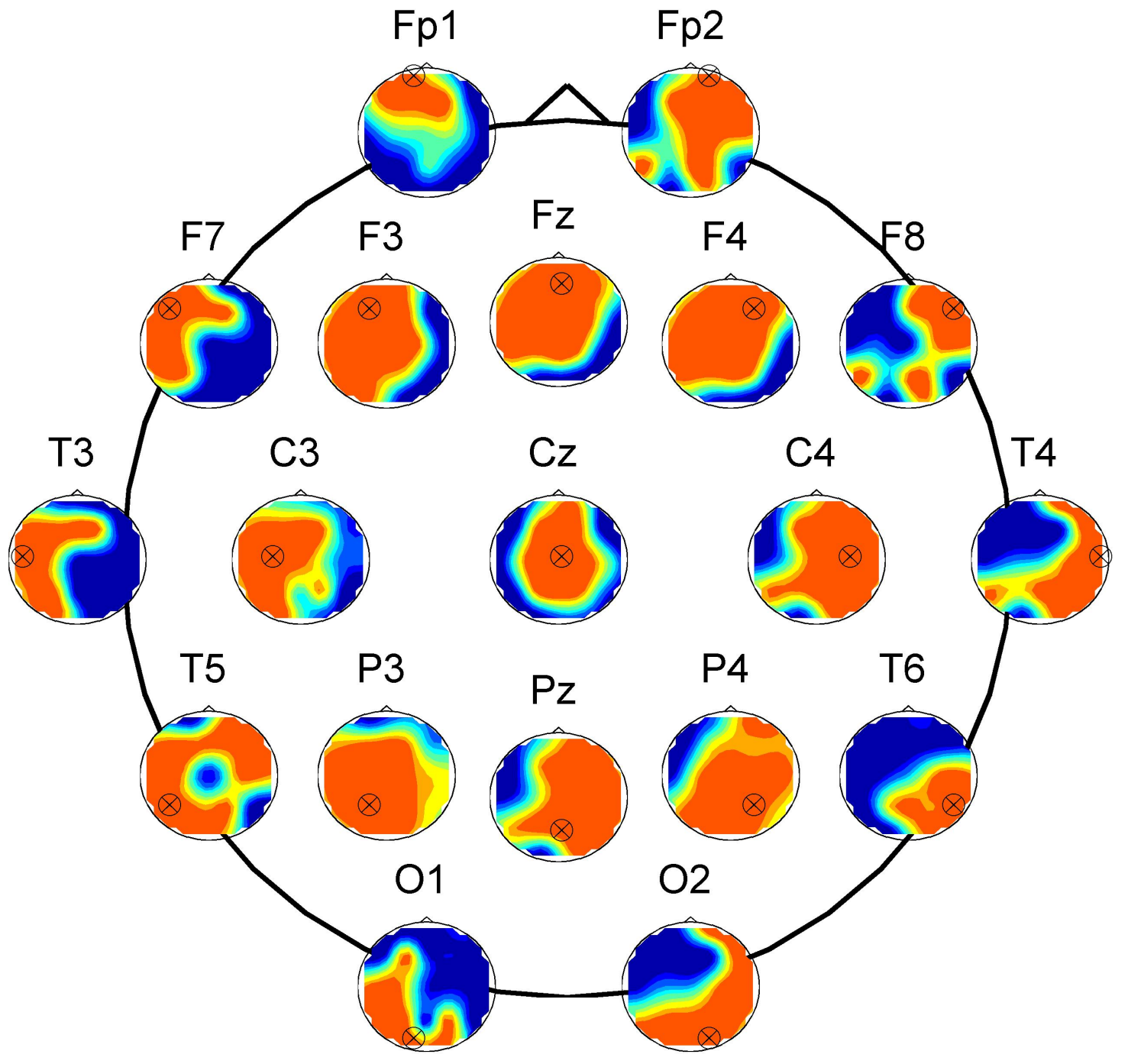

Figure 8: EEG analysis: Heat maps of posterior probabilities of $s_{i j t}=1$ for the induced posterior probabilities of dynamic network connections in $\boldsymbol{\Omega}_{t}$ at snapshot $t=1,501$. Each sub-map shows posterior probabilities that the channel of its plotted location (marked by $\otimes$ ) has a non-zero entry/edge to the other locations, with interpolation across the scalp. See Figure 2 for heat-map scale. 

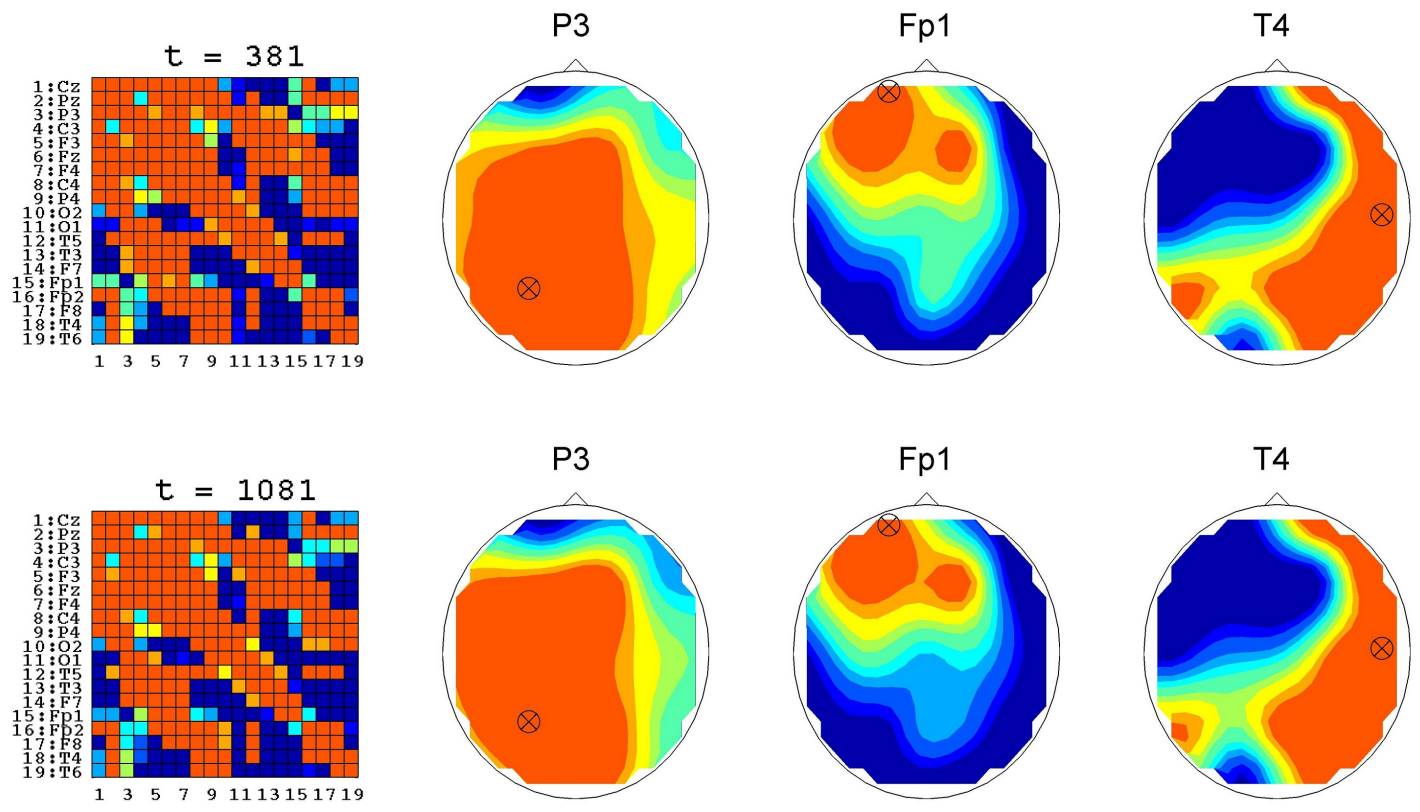

$t=2181$
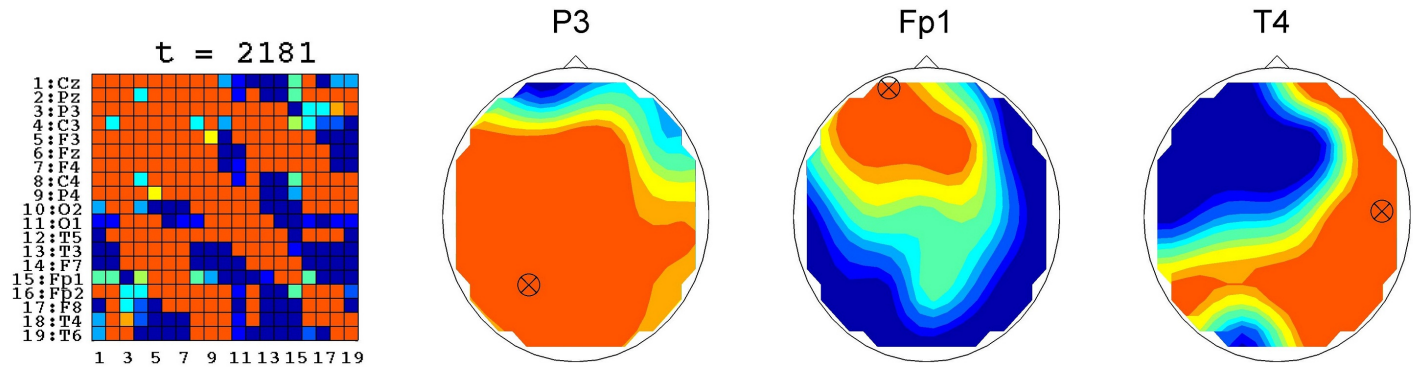

$t=2881$
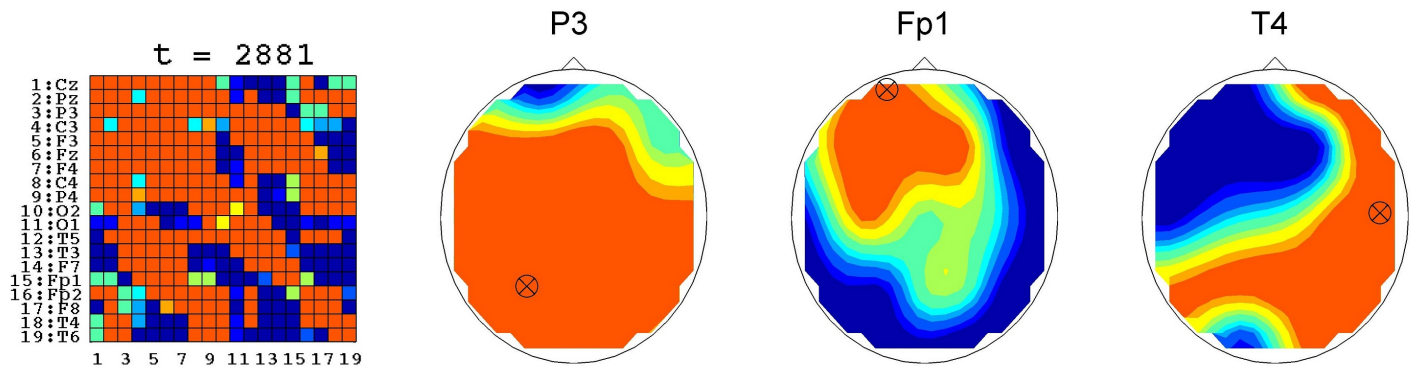

Figure 9: EEG analysis: Insight into the evolution of contemporaneous network connections via posterior probabilities of non-zeros in $\boldsymbol{\Omega}_{t}$, graphed and interpolated as in Figures 7 and 8, now at four selected time points, $t=381,1,081,2,181$ and 2,881, through the course of the signal processing period. 
connected to P3 (the second column) are observed in the left-hand side and central scalp regions in the early period. Then, the area of high probabilities expands to the right-hand side of the scalp towards the later periods. The region of relevant edges linked with Fp1 also expands to the central sites, and the area linked with T4 around the parietal sites changes through the time period. These snapshots show strong evidence of dynamics in some details over time, while the qualitative images of connectivities in the left-hand column of Figure 9 show sustained features in the overall sparsity patterns. It is the case that the quantified strengths of relationships among channels- in terms of posterior estimates of actual values of non-zero elements in $\Omega_{t^{-}}$vary substantially over time, but the existence of connections seems far more stable. This is to be expected: different brain regions have defined/wired pathways of communication to select other regions- the underlying network- while the strengths of communication along these pathways varies as a function of activation.

As Supplemental Material- and as embedded animations in the original technical report version of this paper- we further illustrate and exhibit the above discussion with a video comprising a long series of frames of heat-maps of sparsity probabilities over the full course of the time series. This animates sparsity probabilities over time for the elements of $\boldsymbol{A}_{t}$, the induced connections in $\Omega_{t}$, and selected network sub-maps in terms of interpolated scalp images as in Figure 9 anchored at the three selected channels. This represents the full time-course animation of the evolution of the contemporaneous dynamic network of which Figure 9 provided an initial four snapshots. The stability of structure of the contemporaneous network is quite evident in this animation; again, of course, the actual strengths of relationships (not shown) are substantially time-varying.
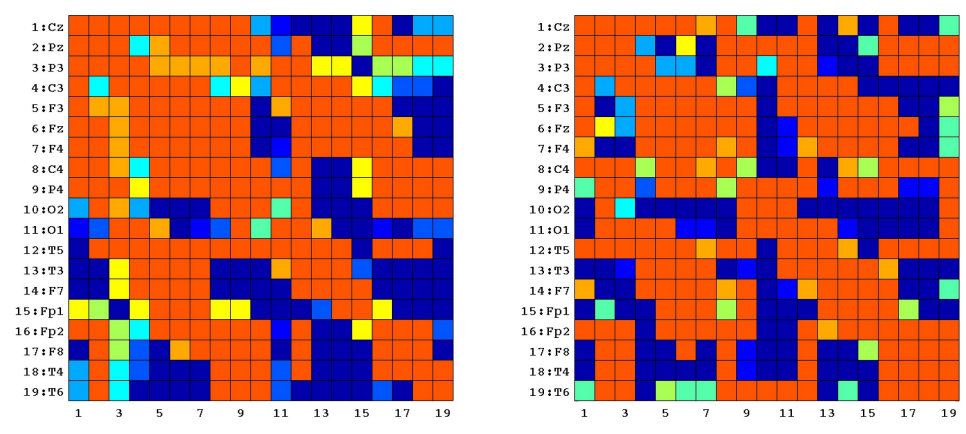

Figure 10: EEG analysis: Posterior probabilities of $s_{i j t}=1$ for induced dynamic network in $\boldsymbol{\Omega}_{t}$ using baseline ordering (left) and factor-based ordering (right), with the layout of probes reordered to match for the comparison. The panels are plotted at snapshot $t=2,001$. See Figure 2 for heat-map scale.

Finally, and as in the FX study, we look at the question of ordering of series, examining an alternative ordering labeled as "factor-based;" see Table 2. Prado et al. (2001) estimate a dynamic factor model for the same EEG time series, providing estimates of factor weights relating the signals of 19 channels to an underlying latent factor process. We refitted our 
model on the series reordered by the estimated posterior means of their factor loadings in that earlier published work. These loadings- or weights- can be regarded as proxies of relations among the 19 channels from that simpler model that assumed a single, underlying "brain seizure process" as a driver of all relationships among channels. Figure 10 exhibits the posterior sparsity probabilities for $\Omega_{t}$ plotted at $t=2,001$, comparing the results from the above baseline analysis with that under this alternative, specific ordering of channels. While there are differences, the overall network patterns are visually very similar to each other. To further illustrate and compare across the two variable orderings, the Supplemental Material includes additional images of sparsity probabilities for $\Omega_{t}$ at four time points spaced across the full period of the time series. There we see strong similarities across time and stability of the overall contemporaneous structure inferred throughout the entire seizure period represented. See supplemental Section C. Additional comments on variable ordering appear in Section 6 below.

\section{Concluding Remarks}

We have illustrated the use of latent threshold modelling in studies of multivariate dynamic networks- empirical networks emerging from multivariate time series models overlaid with dynamically evolving sparsity structure. In the central class of TV-VAR models endowed with a very flexible and efficient Cholesky-type MSV model, latent thresholding enables model fitting with the ability to encourage sparsity in lagged and/or contemporaneous connectivities among variables, and to allow that sparsity structure to be timeand data-adaptive. Our substantive examples, using time series from two quite different contexts, address dynamic relations among responses based on relevance of edges in induced networks, and reveal the utility of the automatically adaptive latent threshold mechanism in analysis of multivariate signal analyses. Dynamic shrinkage- represented in terms of posterior probabilities of zero versus non-zero elements of otherwise timevarying state and volatility matrices- is a key feature of these analyses. Such sparsity probabilities, and their changes in time, provide insights into the nature of the structure of networks. The analysis, based on Bayesian MCMC methods, is computationally efficient and inherently scalable to higher-dimensional time series; the specific class of MSV models based on Cholesky-type decompositions enables decoupling of the overall analysis of an $m$-dimensional time series into $m$ parallel univariate analyses. Recoupling of the posterior Monte Carlo outputs of these $m$ separate analyses then leads to formal Bayesian inference on sparsity structure, dynamics and, if desired, quantified strengths of relationships in terms of posterior inferences on lagged TV-VAR matrices, innovations volatility matrices, and predictive distributions.

There are a number of methodological and computational areas for further investigation. On methodology, one general feature of the Cholesky-style modelling approachwhatever models are specified for time-evolution of the underlying parameter processes- is that it requires an ordering of the time series variables. This is also a feature of the popular parametrization of latent factor models. In our examples, we chose first orderings on sub- 
stantive bases. In the FX study, the ordering chose what we expect to be dominant currencies, with the view that currencies lower in the ordering would be inherently more driven by globally dominant currencies. In the EEG study, we ordered from the central/main vertex channel "out" geographically around the scalp, again based on the substantive notion that the central channels "lead" those further towards the outside. We do see differences in the posteriors based on differing ordering, as we show in the FX study using a reverse ordering. So, while the overall model of equation (1) is order independent, the specific structuring we adopt does make the order a factor in the resulting analysis. How to define a formal statistical approach to determining the order is an open question. Rather than regarding it as a parameter to learn from the data, we regard it as a decision variable to be made as part of the model specification. That then means resulting inferences should be compared across multiple orderings, and this can only be easily done by comparing specific, stylized inferences. In FX studies, for example, we might explore the differences in predictive accuracy and portfolio outcomes, whereas in EEG studies comparisons might be better explored via implied impulse response functions (again a predictive perspective). Clearly, there is much scope here for further research.

On computation, the examples here were run using custom Ox code which is effective and easy to run, but somewhat time consuming. On a 2014 vintage laptop (CPU $8 \times 3.40 \mathrm{GHz}, 16384 \mathrm{MB} \mathrm{ram})$ the FX analysis $(m=20, p=1, T=1,304)$ took just over 7 hours, while the EEG analysis $(m=19, p=2, T=3,000)$ took just over 5 hours. The difference in time demand here comes from that use of the AR(1) stochastic volatility model in the FX study; the discount volatility model used in the EEG analysis leads to substantial computational savings. In terms of scalability, note that the decoupling enabled by the Cholesky-style modelling approach is critical for scaling in number of series $m$, as it enables parallelisation across series. The analysis times reported above were from serial implementation. In higher-order TV-VAR models for large order $p$, the main impact is on the dimension of the state vectors $\boldsymbol{\beta}_{i t}$. The largest of these has length $c=m(p+1)-1$. As the core of the computations involves a Metropolis-Hastings algorithm with conditional normal proposals for these state vectors, then we face computations that scale as $c^{3}$. Evidently, more efficient computational methods will be needed for large $m$ and $p$. The example models here are, however, typical of a lot of current applications, in that $p$ is relatively small even though $m$ may increase; the inherent TV-AR-MSV structure with latent thresholding is able to represent quite complex patterns of temporal inter-relationships among even much higher numbers of series with $p$ in the very small integers. Longer term, one step would be to explore more aggressively exploiting the analysis decoupling feature via distributed CUDA/GPU implementation (Suchard et al. 2010b,a). 


\section{Acknowledgments}

Research was partly supported by grant DMS-1106516 from the National Science Foundation. Any opinions, findings and conclusions or recommendations expressed in this work are those of the authors and do not necessarily reflect the views of the NSF or the Bank of Japan. We are grateful to the Editor-in-Chief, and to three anonymous referees whose positive and constructive suggestions were incorporated in the final version of the paper. We are particularly grateful to one reviewer whose comments on potential economic interpretations of some of the inferred changes over time in network linkages led to improved discussion in the FX study in Section 4.2.

\section{References}

Aguilar, O., Prado, R., Huerta, G., West, M., 1999. Bayesian inference on latent structure in time series (with discussion). In: Bernardo, J. M., Berger, J. O., Dawid, A. P., Smith, A. F. M. (Eds.), Bayesian Statistics 6. Oxford University Press, Oxford, pp. 3-26.

Aguilar, O., West, M., 2000. Bayesian dynamic factor models and portfolio allocation. Journal of Business and Economic Statistics 18, 338-357.

Awokuse, T. O., Chopra, A., Bessler, D. A., 2009. Structural change and international stock market interdependence: Evidence from Asian emerging markets. Economic Modelling 26, 549-559.

BIS, 2010. Triennial Central Bank Survey of Foreign Exchange and Derivatives Market Activity in 2010. Bank for International Settlements.

Carvalho, C. M., West, M., 2007. Dynamic matrix-variate graphical models. Bayesian Analysis 2, 69-98.

Chib, S., Nardari, F., Shephard, N., 2006. Analysis of high dimensional multivariate stochastic volatility models. Journal of Econometrics 134, 341-371.

Dobra, A., Hans, C., Jones, B., Nevins, J. R., Yao, G., West, M., 2004. Sparse graphical models for exploring gene expression data. Journal of Multivariate Analysis 90, 196212.

Doornik, J., 2006. Ox: Object Oriented Matrix Programming. Timberlake Consultants Press, London.

Fujita, A., Sato, J. R., Garay-Malpartida, H. M., Morettin, P. A., Sogayar, M. C., Ferreira, C. E., 2007. Time-varying modeling of gene expression regulatory networks using the wavelet dynamic vector autoregressive method. Bioinformatics 23, 1623-1630.

Granger, C. W. J., 1969. Investigating causal relations by econometric models and crossspectral methods. Econometrica 37, 424-438. 
Jacquier, E., Polson, N. G., Rossi, P. E., 1994. Bayesian analysis of stochastic volatility models. Journal of Business and Economic Statistics 12, 371-389.

Jones, B., Carvalho, C. M., Dobra, A., Hans, C., Carter, C., West, M., 2005. Experiments in stochastic computation for high-dimensional graphical models. Statistical Science 20, 388-400.

Kim, S., Shephard, N., Chib, S., 1998. Stochastic volatility: likelihood inference and comparison with ARCH models. Review of Economic Studies 65, 361-393.

Kitagawa, G., Gersch, W., 1996. Smoothness priors analysis of time series. Lecture Notes in Statistics, Vol.116. Springer-Verlag, New York.

Koop, G., Korobilis, D., 2013. Large time-varying parameter vars. Journal of Econometrics 177, 185-198.

Lauritzen, S. L., 1996. Graphical Models. Clarendon Press, Oxford.

Lopes, H. F., Carvalho, C. M., 2007. Factor stochastic volatility with time varying loadings and Markov switching regimes. Journal of Statistical Planning and Inference 137, 30823091.

Lopes, H. F., McCulloch, R. E., Tsay, R., 2012. Cholesky stochastic volatility models for high-dimensional time series. Tech. rep., University of Chicago, Booth Business School.

Nakajima, J., West, M., 2013a. Bayesian analysis of latent threshold dynamic models. Journal of Business \& Economic Statistics 31, 151-164.

Nakajima, J., West, M., 2013b. Bayesian dynamic factor models: Latent threshold approach. Journal of Financial Econometrics 11, 116-153.

Omori, Y., Chib, S., Shephard, N., Nakajima, J., 2007. Stochastic volatility with leverage: Fast likelihood inference. Journal of Econometrics 140, 425-449.

Onnela, J.-P., Kaski, K., Kertesz, J., 2004. Clustering and information in correlation based financial networks. European Physical Journal B 38, 353-362.

Pinheiro, J. C., Bates, D. M., 1996. Unconstrained parametrizations for variance-covariance matrices. Statistics and Computing 6, 289-296.

Pitt, M. K., Shephard, N., 1999. Time varying covariances: A factor stochastic volatility approach (with discussion). In: Bernardo, J. M., Berger, J. O., Dawid, A. P., Smith, A. F. M. (Eds.), Bayesian Statistics 6. Oxford University Press, Oxford, pp. 547-570.

Prado, R., 1998. Latent structure in non-stationary time series. Ph.D. thesis, Duke University, Durham, N.C. 
Prado, R., 2010a. Characterization of latent structure in brain signals. In: Chow, S., Ferrer, E., Hsieh, F. (Eds.), Statistical Methods for Modeling Human Dynamics. Routledge, Taylor and Francis, New York, pp. 123-153.

Prado, R., 2010b. Multi-state models for mental fatigue. In: O'Hagan, A., West, M. (Eds.), The Handbook of Applied Bayesian Analysis. Oxford University Press, pp. 845-874.

Prado, R., West, M., 2010. Time Series Modeling, Computation, and Inference. Chapman \& Hall/CRC, New York.

Prado, R., West, M., Krystal, A. D., 2001. Multichannel electroencephalographic analyses via dynamic regression models with time-varying lag-lead structure. Journal of the Royal Statistical Society, Series C 50, 95-109.

Primiceri, G. E., 2005. Time varying structural vector autoregressions and monetary policy. Review of Economic Studies 72, 821-852.

Quintana, J. M., Carvalho, C. M., Scott, J., Costigliola, T., 2010. Futures markets, Bayesian forecasting and risk modeling. In: O'Hagan, A., West, M. (Eds.), The Handbook of Applied Bayesian Analysis. Oxford University Press, Oxford, pp. 343-365.

Shephard, N., Pitt, M., 1997. Likelihood analysis of non-Gaussian measurement time series. Biometrika 84, 653-667.

Smith, M., Kohn, R., 2002. Parsimonious covariance matrix estimation for longitudinal data. Journal of the American Statistical Association 97, 1141-1153.

Suchard, M. A., Holmes, C., West, M., 2010a. Some of the what?, why?, how?, who? and where? of graphics processing unit computing for Bayesian analysis. Bulletin of the International Society for Bayesian Analysis 17, 12-16.

Suchard, M. A., Wang, Q., Chan, C., Frelinger, J., Cron, A. J., West, M., 2010b. Understanding GPU programming for statistical computation: Studies in massively parallel massive mixtures. Journal of Computational and Graphical Statistics 19, 419-438.

Tse, C. K., Liu, J., Lau, F. C. M., 2010. A network perspective of the stock market. Journal of Empirical Finance 17, 659-667.

Valdés-Sosa, P. A., 2004. Spatio-temporal autoregressive models defined over brain manifolds. Neuroinformatics 2, 239-250.

Valdés-Sosa, P. A., Sánchez-Bornot, J. M., Lage-Castellanos, A., Vega-Hernández, M., Bosch-Bayard, J., Melie-García, L., Canales-Rodríguez, E., 2005. Estimating brain functional connectivity with sparse multivariate autoregression. Philosophical Transactions of the Royal Society B 360, 969-981. 
Vandewalle, N., Brisbois, F., Tordoir, X., 2001. Self-organized critical topology of stock markets. Quantitative Finance 1, 372-375.

Wang, H., 2010. Sparse seemingly unrelated regression modelling: Applications in finance and econometrics. Computational Statistics and Data Analysis 54, 2866-2877.

Wang, H., West, M., 2009. Bayesian analysis of matrix normal graphical models. Biometrika 96, 821-834.

Watanabe, T., Omori, Y., 2004. A multi-move sampler for estimating non-Gaussian time series models: Comments on Shephard and Pitt (1997). Biometrika 91, 246-248.

Weiner, R. D., Krystal, A. D., 1994. The present use of electroconvulsive therapy. Annual Review of Medicine 45, 273-281.

West, M., 2013. Bayesian dynamic modelling. In: Damien, P., Dellaportes, P., Polson, N. G., Stephens, D. A. (Eds.), Bayesian Theory and Applications. Clarendon: Oxford University Press, Ch. 8, pp. 145-166.

West, M., Harrison, P. J., 1997. Bayesian Forecasting and Dynamic Models, 2nd Edition. Springer-Verlag, New York.

West, M., Prado, R., Krystal, A. D., 1999. Evaluation and comparison of EEG traces: latent structure in nonstationary time series. Journal of the American Statistical Association 94, 375-387.

Whittaker, J., 1990. Graphical Models in Applied Multivariate Statistics. John Wiley and Sons, Chichester, United Kingdom.

Yoshida, R., West, M., 2010. Bayesian learning in sparse graphical factor models via annealed entropy. Journal of Machine Learning Research 11, 1771-1798.

Zhou, X., Nakajima, J., West, M., 2014. Bayesian forecasting and portfolio decisions using dynamic dependent sparse factor sparse. International Journal of Forecasting 30, 963980 . 


\section{In Memory of Bill Fitzgerald}

The work presented here- flexible, practically motivated dynamic models in Bayesian signal processing, fitted using MCMC methods, and showcased in important applicationslinks intimately to central themes in the work of Bill Fitzgerald. We dedicate the paper to Bill's memory. Bill was a true pioneer at the interfaces of Bayesian statistics and traditional engineering signal processing, and had a major influence and impact on the very positive cross-breeding between communities that we have experienced for over two decades. Bill was a personable colleague and professional leader with a vision for the disciplines and cross-disciplinary integration; and, of course, a delightful and engaging individual and friend. Bill's impact on modern signal processing and applied statistical science- through his own work and that with colleagues and students- was enormous, and his legacy will live on through his wide-ranging writing, and through the continuing work of those he mentored and influenced.

Author information

Jouchi Nakajima (sites.google.com/site/jnakajimaweb/) is Economist and Deputy Director of the Monetary Affairs Department at the Bank of Japan, Tokyo. His main research foci are in Bayesian time series analysis, dynamic modelling and methods for econometrics, policy studies and finance; he has published widely in these areas. Jouchi received his MA in Economics at the University of Tokyo, followed by MS and PhD degrees in Statistical Science at Duke University.

Mike West (www.stat.duke.edu/ $/ \mathrm{mw}$ ) is The Arts \& Sciences Professor of Statistics \& Decision Sciences in the Department of Statistical Science at Duke University. Mike led development of the department from 1990 to 2001, has served in the establishment- and as board member- of several national research institutes and companies, and is past President of the International Society for Bayesian Analysis. Mike works in theory and applications of Bayesian statistics, with highlights in dynamic modelling, time series analysis and forecasting. Mike has advised nearly $60 \mathrm{PhD}$ students and postdoctoral associates, and numerous undergraduate and MS students. 